
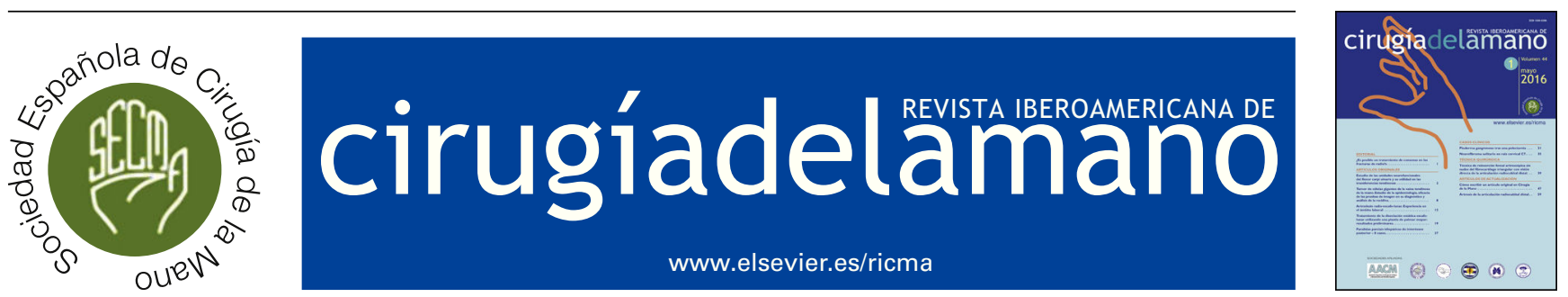

ARTÍCULO ORIGINAL

\title{
Modificación simplificada de la técnica de Zancolli para la corrección de la garra cubital
}

\section{R. Monreal González ${ }^{\text {a,* }}$, D. Gómez Hernández ${ }^{\mathrm{b}}$, E. Faedo Castro ${ }^{\mathrm{a}}$ y G. Osinaga Egüez ${ }^{\mathrm{c}}$}

a Centro Médico MEDEX, San Isidro, Lima, Perú

${ }^{\mathrm{b}}$ Hospital Universitario Madrid Torrelodones, Torrelodones, Madrid, España

' Hospital Obrero N. ${ }^{\circ}$ 3, Caja Nacional de Salud, Santa Cruz, Bolivia

Recibido el 25 de abril de 2016; aceptado el 26 de agosto de 2016

Disponible en Internet el 4 de octubre de 2016

\section{PALABRAS CLAVE \\ Garra cubital; \\ Parálisis; \\ Vaina tendinosa; \\ Corrección quirúrgica}

\section{KEYWORDS}

Ulnar claw;

Paralysis;

Tendon sheath;

Surgical correction

\begin{abstract}
Resumen
Objetivos: Estudiar los resultados obtenidos con la técnica de Chevallard, versión simplificada de la técnica de Zancolli, para corregir la deformidad de la mano en garra secundaria a una parálisis cubital.

Material y método: Estudio retrospectivo (enero 2006-diciembre 2013) de 16 pacientes (16 manos) que fueron intervenidos quirúrgicamente por deformidad en garra de la mano secundaria a parálisis del nervio cubital. Siguiendo los criterios de Brand, los pacientes fueron evaluados a las 6 semanas, 3 meses, 6 meses y al año.

Resultados: Los resultados al año de la intervención fueron: excelentes en el 18,75\% ( 3 pacientes); buenos en el 75\% (12 pacientes) y malos en el 6,25\% (un paciente). Este último requirió una nueva intervención para solucionar la recidiva de la garra.

Conclusión: La técnica simplificada de Zancolli, o técnica de Chevallard, permite corregir fácilmente la deformidad de la mano en garra secundaria a una parálisis cubital, no hipotecando una ulterior intervención en el caso de recidiva.

(c) 2016 SECMA. Publicado por Elsevier España, S.L.U. Este es un artículo Open Access bajo la licencia CC BY-NC-ND (http://creativecommons.org/licenses/by-nc-nd/4.0/).
\end{abstract}

Simplified modification of the Zancolli technique for the correction of the ulnar claw

\section{Abstract}

Objectives: The objective of this paper is to study the results obtained with the Chevallard technique (simplified version of the Zancolli technique), to correct claw hand deformity, secondary to ulnar nerve palsy.

\footnotetext{
* Autor para correspondencia.

Correo electrónico: rmonreal59@yahoo.es (R. Monreal González).
} 
Materials and method: Retrospective study conducted on 16 patients (16 hands) who underwent claw hand correction (January 2006-December 2013), and were evaluated at the 6 weeks, 3 months, 6 months and one year.

Results: At one year later, an objective assessment (Brand criteria) showed excellent results in $18.75 \%$ ( 3 patients), good in $75 \%$ (12 patients), and poor in $6.25 \%$ (1 patient). The latter required a new intervention to solve the recurrence of the claw.

Conclusion: The simplified of Zancolli technique or technique of Chevallard allows to easily correct the ulnar claw hand deformity, not ruling out a further intervention in the case of recurrence.

(C) 2016 SECMA. Published by Elsevier España, S.L.U. This is an open access article under the CC BY-NC-ND license (http://creativecommons.org/licenses/by-nc-nd/4.0/).

\section{Introducción}

La parálisis de los músculos intrínsecos de la mano da lugar a la deformidad denominada «mano en garra», la cual puede ser la manifestación de diversas entidades patológicas como el ictus, la parálisis cerebral, la enfermedad de Charcot-Marie-Tooth, la distrofia muscular, la lepra, los traumatismos, la hernia discal cervical, y/o las neuropatías por compresión ${ }^{1}$. Quizás la mano en garra más famosa fue la de Miguel de Cervantes Saavedra, quien en octubre de 1571 recibió 3 heridas por arma de fuego en la batalla de Lepanto, de las que la más grave afectó su mano izquierda ${ }^{2}$. Sin embargo, la deformidad del gran exponente de la literatura española fue el resultado de una compleja lesión en la cual se afectaron varias estructuras anatómicas (tendones, nervios, huesos, nervios y músculos).

El principio básico de la corrección de la mano en garra consiste en sustituir la función de los músculos lumbricales e interóseos, responsables de la acción sinérgica de la flexión de las articulaciones metacarpofalángicas (MCF) y extensión de las articulaciones interfalángicas (IF).

El objetivo de este trabajo es el de dar a conocer los resultados obtenidos en una serie de pacientes con mano en garra secundaria a parálisis del nervio cubital, que fueron tratados con la técnica descrita en 1987 por Alexis J. Chevallard $^{3}$, una versión simplificada de la técnica de Zancolli publicada en $1957^{4}$. No hemos encontrado en la literatura revisada otro reporte de los resultados de esta técnica quirúrgica.

\section{Material y método}

Durante el periodo comprendido entre enero de 2006 y diciembre de 2016, fueron intervenidos quirúrgicamente 16 pacientes (16 manos) con deformidad en garra de la mano secundaria a una parálisis cubital. La edad promedio fue 32,6 años y el tiempo promedio transcurrido entre el momento de la lesión y la cirugía fue de 9,1 meses. Otros datos de la serie estudiada se muestran en la tabla 1.

Los criterios de inclusión pueden observarse en la tabla 2. Como criterios de evaluación de los resultados se siguieron los de la clasificación de Brand, mostrados en la tabla 3.

Después de la operación los pacientes fueron evaluados clínicamente a las 6 semanas, 3 meses, 6 meses y al año. El resultado final de la corrección de la deformidad y el estado funcional de la mano corresponden a la evaluación realizada al año de la cirugía.

\section{Técnica quirúrgica}

La cirugía se realiza bajo anestesia general. Con el paciente en decúbito supino e isquemia preventiva con manguito neumático en el miembro superior indicado, se practica una incisión transversal a nivel del pliegue palmar distal de la mano. En el caso de que la deformidad interese los dedos $2 .^{\circ}-5 .^{\circ}$, la incisión se extiende desde el borde radial al borde cubital, mientras que si la deformidad afecta solamente los dedos $4 .^{\circ}$ y $5 .^{\circ}$, basta una incisión más pequeña en el lado cubital. A continuación se exponen las vainas flexoras a nivel de las poleas $A 1$ y A2. Después de practicar una pequeña ventana de la vaina tendinosa en forma de T distal a la polea A1 (modificación de la técnica original), se identifica y expone el tendón flexor superficial del dedo seleccionado, el cual es suturado directamente al borde proximal de la polea A2 con un par de puntos de prolene $3 / 0$, previa realización de una hiperextensión pasiva de las falanges (fig. 1). Después del cierre de la piel con prolene $5 / 0$, se procede a inmovilizar la mano y muñeca con una férula de yeso, manteniendo las articulaciones MCF a $90^{\circ}$ y las IF en extensión, pero permitiendo su flexión libre. A las 2 semanas se hace un cambio de férula y se retiran los puntos y, a las 4 semanas, se deja completamente libre la extremidad para la movilidad activa de la muñeca y los dedos. No se requiere ningún tipo de rehabilitación.

\section{Resultados}

Al año de la intervención, los resultados obtenidos según la clasificación de Brand ${ }^{5}$ fueron: excelente en 3 (18,75\%) (fig. 2), bueno en 12 (75\%) y malo en uno (6,25\%) (tabla 1). Este último requirió una reintervención con otra técnica, debido a la persistencia de la garra.

\section{Discusión}

La mano en garra, también conocida como intrinsecus minus, es una deformidad resultante de traumatismos del nervio cubital o de ciertas enfermedades neurológicas que 
Tabla 1 Datos generales

\begin{tabular}{|c|c|c|c|c|c|c|c|c|}
\hline N. ${ }^{\circ}$ & $\begin{array}{l}\text { Edad } \\
\text { (años) }\end{array}$ & Género & Etiología & $\begin{array}{l}\text { Nivel de } \\
\text { lesión }\end{array}$ & EMG & Cirugía previa & $\begin{array}{l}\text { Tiempo de } \\
\text { evolución } \\
\text { preoperatorio } \\
\text { (meses) }\end{array}$ & $\begin{array}{l}\text { Evaluación } \\
\text { funcional }\end{array}$ \\
\hline 1 & 18 & M & Herida de muñeca & Baja & $x$ & Neurorrafia & 6 & $E$ \\
\hline 2 & 31 & M & $\begin{array}{l}\text { Fractura expuesta } \\
\text { de antebrazo }\end{array}$ & Baja & $x$ & $\begin{array}{l}\text { Osteosíntesis + } \\
\text { neurorrafia }\end{array}$ & 10 & B \\
\hline 3 & 28 & M & Herida de muñeca & Baja & $x$ & Injerto nervioso & 14 & $E$ \\
\hline 4 & 19 & M & $\begin{array}{l}\text { Lesión del plexo } \\
\text { braquial }\end{array}$ & Alta & $x$ & $\begin{array}{l}\text { Transferencia } \\
\text { tendinosa } \\
\text { latero-lateral }\end{array}$ & 21 & B \\
\hline 5 & 41 & M & $\begin{array}{l}\text { Fractura-luxación } \\
\text { del codo }\end{array}$ & Baja & $x$ & Osteosíntesis & 4 & M \\
\hline 6 & 54 & M & $\begin{array}{l}\text { Fractura de } \\
\text { antebrazo }\end{array}$ & Baja & $x$ & Osteosíntesis & 8 & B \\
\hline 7 & 38 & $\mathrm{~F}$ & $\begin{array}{l}\text { Herida de } \\
\text { antebrazo }\end{array}$ & Baja & $x$ & & 6 & B \\
\hline 8 & 21 & M & Herida de brazo & Alta & $x$ & $\begin{array}{l}\text { Injerto nervioso } \\
\text { Transferencia } \\
\text { tendinosa } \\
\text { latero-lateral }\end{array}$ & 7 & B \\
\hline 9 & 43 & M & $\begin{array}{l}\text { Síndrome del túnel } \\
\text { cubital }\end{array}$ & Baja & $\mathrm{x}$ & $\begin{array}{l}\text { Liberación del } \\
\text { nervio cubital }\end{array}$ & 16 & $E$ \\
\hline 10 & 23 & $\mathrm{~F}$ & $\begin{array}{l}\text { Neuroma } \\
\text { intraneural }\end{array}$ & Baja & $x$ & $\begin{array}{l}\text { Resección } \\
\text { tumoral e } \\
\text { injerto } \\
\text { nervioso }\end{array}$ & 8 & B \\
\hline 11 & 37 & M & Herida de brazo & Alta & $x$ & Neurorrafia & 5 & B \\
\hline 12 & 44 & M & $\begin{array}{l}\text { Luxación expuesta } \\
\text { de codo }\end{array}$ & Baja & $\mathrm{x}$ & & 4 & B \\
\hline 13 & 30 & M & Herida de muñeca & Baja & $x$ & Neurorrafia & 3 & B \\
\hline 14 & 21 & M & $\begin{array}{l}\text { Lesión del plexo } \\
\text { braquial }\end{array}$ & Alta & $x$ & $\begin{array}{l}\text { Transferencia } \\
\text { tendinosa } \\
\text { latero-lateral }\end{array}$ & 18 & B \\
\hline 15 & 39 & $\mathrm{~F}$ & Herida de muñeca & Baja & $x$ & & 9 & $\mathrm{E}$ \\
\hline 16 & 35 & M & $\begin{array}{l}\text { Fractura expuesta } \\
\text { de cúbito }\end{array}$ & Alta & $x$ & Osteosíntesis & 7 & B \\
\hline
\end{tabular}

Tabla 2 Criterios de inclusión

1. Garra simple de los dedos secundaria a lesión del nervio cubital aislada no asociada a lesiones de otros nervios periféricos, lesión nerviosa central (medular o encefálica) o como secuela de síndrome compartimental

2. Deformidades articulares fijas o contracturas de la piel

3. Ausencia de focos infecciosos

4. Maniobra de Bouvier positiva (al bloquear la hiperextensión de las articulaciones metacarpofalángicas se logra extender las articulaciones interfalángicas)

se caracterizan por la hiperextensión de las articulaciones MCF y flexión de las IF, tanto proximales (IFP) como distales (IFD) ${ }^{1}$. Si bien la parálisis afecta predominantemente los dedos meñique y anular, puede ser que solo afecte al dedo meñique, ya que el lumbrical del dedo anular tiene una inervación dual con el nervio mediano en el $50 \%$ de la población.

Sin embargo, el nivel en el que se encuentra la lesión nerviosa es el que nos va a determinar la presentación clínica de la garra y el tratamiento a seguir. Así por ejemplo, en las parálisis altas, es decir, por encima de las ramas del flexor carpi ulnaris y de los flexores profundos de los dedos meñique y anular, la parálisis afecta los flexores profundos de los citados dedos y, en consecuencia, la deformidad en garra no es tan acusada como la observada en las parálisis cubitales bajas. Sin embargo, tanto en uno como en otro caso, la laxitud de las articulaciones MCF adquiere una especial relevancia en la severidad de la garra. Aunque la extensión de las articulaciones IF se produce principalmente a través de la musculatura intrínseca, los músculos 
Tabla 3 Evaluación funcional según los criterios de Brand

\begin{tabular}{ll}
\hline Excelente & El paciente puede abrir la mano manteniendo extendida todas las articulaciones metacarpofalángicas e \\
& interfalángicas, puede flexionar las articulaciones metacarpofalángicas a $90^{\circ}$, manteniendo recta la \\
& articulación interfalángica. Dispone de un mecanismo normal de cierre del puño, la fuerza de agarre y \\
la función de la mano son casi normales & \\
Bueno & El paciente puede extender sus dedos, por lo menos hasta $150^{\circ}$ a nivel de la articulación interfalángica \\
& y metacarpofalángica. El mecanismo de cierre muestra que la articulación metacarpofalángica puede \\
Fallido & ser flexionada $90^{\circ}$ antes que las articulaciones interfalángicas comiencen a flexionarse \\
& Las deformidades en garra de los dedos mejoraron su aspecto en comparación con la etapa \\
& preoperativa pero el paciente es incapaz de extender las articulaciones interfalángicas lo suficiente \\
como para agarrar el vaso & \\
Malo & Cualquier mano en la que no se corrige la deformidad ni mejora la función
\end{tabular}

Fuente: Brand ${ }^{5}$.

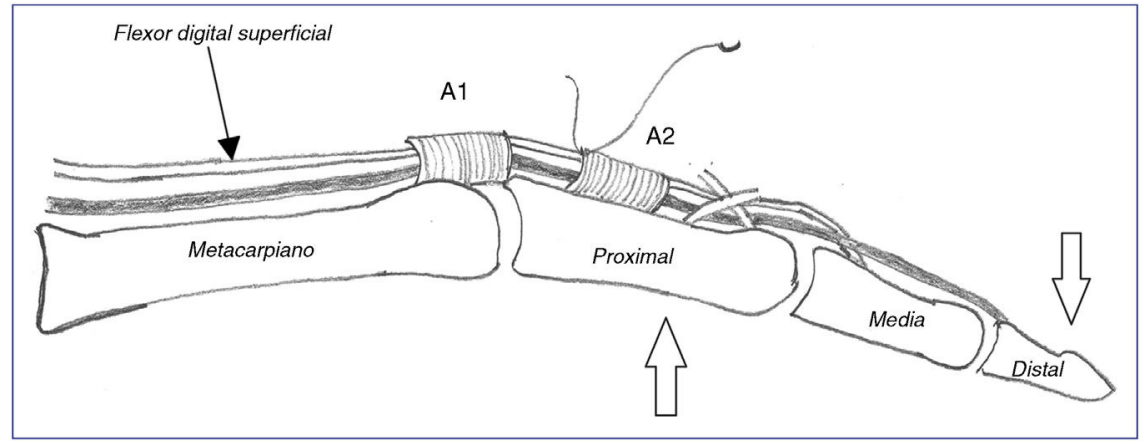

Figura 1 Técnica simplificada de Zancolli o técnica de Chevallard. El tendón flexor superficial del dedo seleccionado es suturado directamente al borde proximal de la polea A2 (sin desinserción) previa realización de una hiperextensión pasiva del dedo.
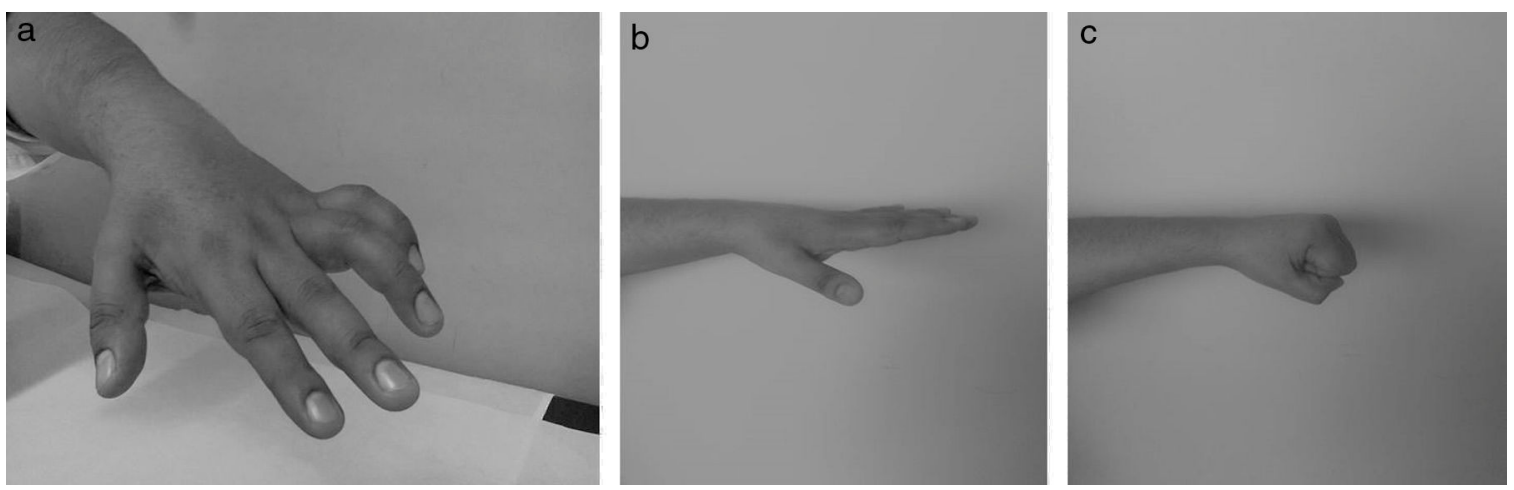

Figura 2 Deformidad en garra de la mano corregida satisfactoriamente con la técnica de Chevallard. a) Imagen clínica preoperatoria. b) Imagen clínica lateral postoperatoria con los dedos extendidos. c) Imagen clínica lateral postoperatoria con los dedos en flexión.

extrínsecos son también capaces de extenderlas siempre y cuando las articulaciones MCF no se encuentren en hiperextensión y el mecanismo extensor de las articulaciones IFP no sea demasiado laxo. Si las articulaciones MCF se extienden demasiado, la musculatura extrínseca se encuentra al máximo de su excursión y no es capaz de transmitir, a través del deslizamiento de la bandeleta central, la fuerza necesaria para extender las articulaciones IFP. Si, por el contrario, se evita la hiperextensión de las articulaciones MCF, los extensores extrínsecos sí son capaces de extender las articulaciones IF, maniobra conocida como de Bouvier ${ }^{6}$ (fig. 3).
En cuanto al tratamiento se refiere, existen multitud de procedimientos quirúrgicos para corregir la mano en garra, todos ellos encaminados a facilitar la «cascada de flexión» de los dedos, es decir, la flexión de las articulaciones MCF antes de que se produzca la flexión de las articulaciones IF. En general se dividen en estáticos o dinámicos ${ }^{1}$. Dentro de los procedimientos estáticos, aparte de la artrodesis MCF (totalmente olvidada), se encuentran la tenodesis ${ }^{7-11}$ y la capsulodesis de la placa palmar ${ }^{4}$. La principal desventaja de estas técnicas es que no pueden recuperar la debilidad muscular y la recidiva de la garra es frecuente. 

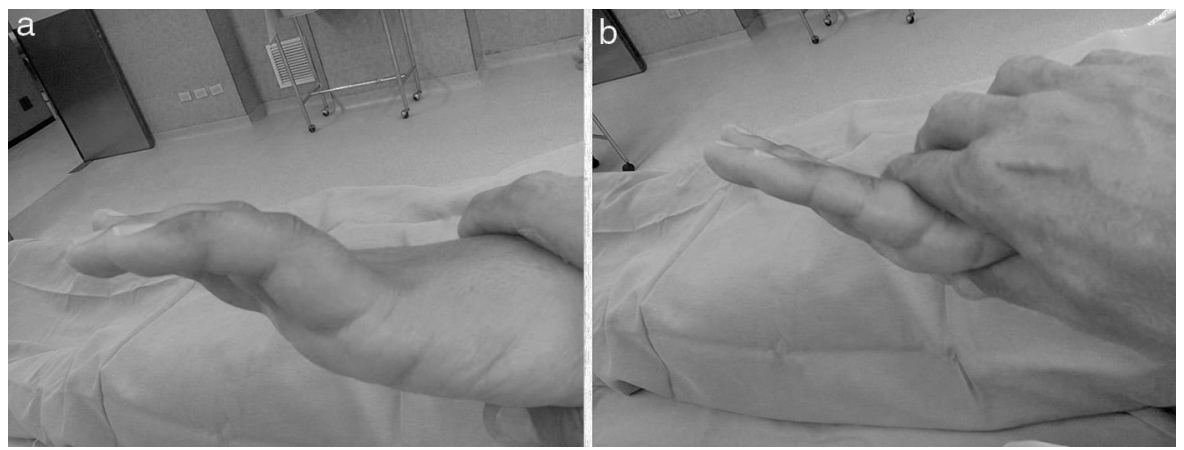

Figura 3 Maniobra de Bouvier. a) Posición inicial de la mano. b) Al prevenir la hiperextensión de la articulaciones MCF por presión dorsal sobre las falanges proximales, se logra la extensión completa de las articulaciones IF.

En cuanto a los procedimientos dinámicos, se encuentran las transferencias tendinosas que, aunque implican el sacrificio de una unidad músculo-tendón para suplir la parálisis de otra unidad, presentan la ventaja que corrigen muy bien la garra, mejoran la fuerza de presión y la sincronización normal de la flexión de los dedos. Dentro de estas técnicas, se encuentra la denominada «lazo de Zancolli» ${ }^{12}$, consistente en seccionar los flexores superficiales de los dedos afectados entre las poleas A1 y A2, y suturarlos sobre sí mismos, a nivel proximal de la polea $\mathrm{A} 1$. El inconveniente de esta técnica es que si existe una laxitud ligamentosa a nivel de la articulación IFP, y la sección del flexor superficial puede dar lugar a una deformidad en cuello de cisne debido a la ausencia de oposición de los extensores extrínsecos. La técnica descrita en este artículo, o técnica de Chevallard, evita tal complicación y, además, no sacrifica ninguna estructura anatómica para suplir otra paralizada. De los 30 casos de parálisis publicados por Chevallard, la garra se corrigió en 25 casos, parcialmente en 3 casos y en 2 casos se requirió otra intervención por recidiva ${ }^{3}$, resultados muy parecidos a los observados en nuestra serie.

La movilización postoperatoria inmediata que aplicamos en todos los pacientes tiene su fundamentación en estudios previos que demuestran que la movilización inmediata es segura y mejora el rango de movilidad activa de la flexión digital, a diferencia de la inmovilización prolongada $^{13}$.

Las limitaciones de este estudio son su carácter retrospectivo y que la serie consta de pocos casos. Sin embargo, dada la fácil ejecución de la técnica y que no requiere una rehabilitación específica, creemos que puede ser tenida en cuenta a la hora de abordar esta compleja deformidad, como es la deformidad en garra. Quizás sería muy útil realizar un estudio prospectivo en que se tengan en cuenta otros factores como la edad, el sexo, la duración de la parálisis, el grado de contractura y la experiencia del cirujano.

\section{Responsabilidades éticas}

Protección de personas y animales. Los autores declaran que los procedimientos seguidos se conformaron a las normas éticas del comité de experimentación humana responsable y de acuerdo con la Asociación Médica Mundial y la Declaración de Helsinki.

Confidencialidad de los datos. Los autores declaran que han seguido los protocolos de su centro de trabajo sobre la publicación de datos de pacientes.

Derecho a la privacidad y consentimiento informado. Los autores han obtenido el consentimiento informado de los pacientes y/o sujetos referidos en el artículo. Este documento obra en poder del autor de correspondencia.

\section{Conflicto de intereses}

Los autores declaran no tener ningún conflicto de intereses.

\section{Bibliografía}

1. Sapienza A, Green S. Correction of the claw hand. Hand Clin. 2012;28:53-66.

2. López Alonso A. Cervantes, manco y bien manco. Alcalá de Henares: Servicio de Publicaciones, Universidad de Alcalá de Henares; 1997. 225 pp.

3. Chevallard AJ. Simplified surgical technique for flexible clawed hand rehabilitation. Int J Leprosy. 1987;55:160-2.

4. Zancolli E. Corrección de la garra digital por parálisis intrínseca; la operación del lazo. Acta Ortop Latinoam. 1974;1: 65-71.

5. Brand PW. Paralytic claw hand with special reference to paralysis in leprosy and treatment by the sublimis transfer of Stiles and Bunnell. J Bone Joint Surg Br. 1958;40: 618-32.

6. Bouvier SHV. Note sur une paralysie partielle des muscles de la main. Bull Acad Nat Med (Paris). 1851;18:125-39.

7. Riordan DC. Tendon transplantation in median nerve and ulnar nerve paralysis. J Bone Joint Surg Am. 1953;35: 312-20.

8. Riordan DC. Tendon transfers for median, ulnar or radial nerve palsy. Hand. 1969;1:42-6.

9. Srinivasan $\mathrm{H}$. The extensor diversion graft operation for correction of intrinsic minus fingers in leprosy. J Bone Joint Surg Br. 1973;55:58-65.

10. Smith RJ. Metacarpal sling tenodesis. Bull Hosp Joint Dis. 1984;44:466-9. 
11. Ozkan T, Ozer K, Ayan Gulgonen A. Three tendon transfer methods in reconstruction of ulnar nerve palsy. J Hand Surg Am. 2003;28:35-43.

12. Zancolli EA. Claw hand caused by paralysis of the intrinsic muscles. A simple surgical procedure for its correction. J Bone Joint Surg Am. 1957;39:1076-80.
13. Santosh R. Immediate postoperative active mobilization versus immobilization following tendon transfer for claw deformity correction in the hand. J Hand Surg Am. 2008;33: 232-40. 